PROCEEDINGS OF THE

AMERICAN MATHEMATICAL SOCIETY

Volume 126, Number 10, October 1998, Pages 3019-3026

S $0002-9939(98) 04547-\mathrm{X}$

\title{
ON COMPLEMENTARY SUBSPACES OF HILBERT SPACE
}

\author{
W. E. LONGSTAFF AND ORESTE PANAIA
}

(Communicated by Palle E. T. Jorgensen)

\begin{abstract}
Every pair $\{M, N\}$ of non-trivial topologically complementary subspaces of a Hilbert space is unitarily equivalent to a pair of the form $\{G(-A) \oplus K, G(A) \oplus(0)\}$ on a Hilbert space $H \oplus H \oplus K$. Here $K$ is possibly (0), $A \in \mathcal{B}(H)$ is a positive injective contraction and $G( \pm A)$ denotes the graph of $\pm A$. For such a pair $\{M, N\}$ the following are equivalent: (i) $\{M, N\}$ is similar to a pair in generic position; (ii) $M$ and $N$ have a common algebraic complement; (iii) $\{M, N\}$ is similar to $\{G(X), G(Y)\}$ for some operators $X, Y$ on a Hilbert space. These conditions need not be satisfied. A second example is given (the first due to T. Kato), involving only compact operators, of a double triangle subspace lattice which is not similar to any operator double triangle.
\end{abstract}

\section{INTRODUCTION AND PRELIMINARIES}

Throughout what follows all Hilbert spaces will be over the complex field. The terms 'operator' and 'subspace' will mean 'bounded linear transformation' and 'closed linear manifold', respectively. For an operator $T, \mathcal{R}(T)$ denotes it range and $G(T)$ its graph. For a subspace $K, P_{K}$ denotes the orthogonal projection onto $K$. Two subspaces $M$ and $N$ of a Hilbert space $\mathcal{H}$ are algebraically complementary if $M \cap N=(0)$ and $M+N=\mathcal{H}$; they are topologically complementary if $M \cap N=(0)$ and $M \vee N=\mathcal{H}$, where, as usual, ' $\vee$ ' denotes 'closed linear span'.

Two subspaces $M$ and $N$ are in generic position in $\mathcal{H}$ if $M \cap N=M^{\perp} \cap N^{\perp}=$ $M^{\perp} \cap N=M \cap N^{\perp}=(0)$. Such $M$ and $N$ are topologically complementary. Also, each is non-trivial (if $\mathcal{H} \neq(0)$ ). In [2] Halmos shows that if $M$ and $N$ are in generic position, then the (ordered) pair $\langle M, N\rangle$ is unitarily equivalent to the (ordered) pair $\langle G(-A), G(A)\rangle$ of subspaces of $H \oplus H$, for some Hilbert space $H$ and some positive injective contraction $A \in \mathcal{B}(H)$ with $I-A$ injective. More precisely, this means that there exists a unitary operator from $\mathcal{H}$ onto $H \oplus H$ which maps $M$ and $N$ onto $G(-A)$ and $G(A)$, respectively. We shall continue to use Halmos' pointed bracket notation \langle\rangle to specify order, even for subspace triples, and use simple braces \{\} when no particular order is specified. For simplicity, any surjective isometric operator between Hilbert spaces will be called a unitary operator. In [9] Papadakis defines a pair $\{M, N\}$ to be in generalised generic position in $\mathcal{H}$ if $M \cap N=$ $M^{\perp} \cap N^{\perp}=(0)$ and $\operatorname{dim}\left(M^{\perp} \cap N\right)=\operatorname{dim}\left(M \cap N^{\perp}\right)$, where $\operatorname{dim} L$ denotes the Hilbert dimension of $L$. (Once again, such $M$ and $N$ are toplogically complementary and each is non-trivial.) He observes (without proof) that Halmos' conclusion still

Received by the editors March 14, 1997.

1991 Mathematics Subject Classification. Primary 46C05.

(C)1998 American Mathematical Society 
holds for this (strictly) more general relative positioning, except that the operator $I-A$ can no longer be required to be injective. We show that any topologically complementary pair $\{M, N\}$ of non-trivial subspaces is unitarily equivalent to a pair of the form $\{G(-A) \oplus K, G(A) \oplus(0)\}$ on some Hilbert space $H \oplus H \oplus K$, with $K$ possibly (0) and $A \in \mathcal{B}(H)$ a positive injective contraction. Part of the proof gives a simple proof of Papadakis' observation.

For simplicity, any bicontinuous linear bijection between Hilbert spaces will be called an invertible operator. Two families, $\mathcal{F}_{1}$ and $\mathcal{F}_{2}$, of subspaces of Hilbert spaces $H_{1}$ and $H_{2}$, respectively, will be called similar if there exists an invertible operator $S: H_{1} \rightarrow H_{2}$ such that $S \mathcal{F}_{1}=\mathcal{F}_{2}$ (with a slight abuse of notation). We show directly that a pair of the form $\{G(-A) \oplus K, G(A) \oplus(0)\}$, as above, need not be similar to any pair of the form $\{G(X), G(Y)\}$ where $X, Y \in \mathcal{B}\left(H_{1}\right)$ for some Hilbert space $H_{1}$. Also, using a result of [8], we prove that for a pair $\{M, N\}$ of non-trivial topologically complementary subspaces the following are equivalent: (i) $\{M, N\}$ is similar to a pair in generic position; (ii) $M$ and $N$ have a common algebraic complement; (iii) $\{M, N\}$ is similar to $\{G(X), G(Y)\}$ for some operators $X$ and $Y$. (We also extend this to families of pairwise topologically complementary subspaces.) These results lead to a second example of a double triangle subspace lattice which is not similar to any operator double triangle (for definitions, see below). The first example, described in [8], is due to T. Kato and involves an unbounded linear transformation. Our example involves only compact operators. As usual, by a subspace lattice on a Hilbert space $H$ we mean a family $\mathcal{L}$ of subspaces of $H$ satisfying (i) $(0), H \in \mathcal{L}$, (ii) for every family $\left\{L_{\gamma}\right\}_{\Gamma}$ of elements of $\mathcal{L}, \bigcap_{\gamma \in \Gamma} L_{\gamma} \in \mathcal{L}$ and $\bigvee_{\gamma \in \Gamma} L_{\gamma} \in \mathcal{L}$

\section{Main Results}

The following lemma is stated for convenience. Its proof is an elementary exercise.

Lemma 1. Let $J, K$ and $L$ be subspaces of a Hilbert space $H$. Then

(i) if $K \subseteq J$, then $J \cap(K+L)=K+(J \cap L)$,

(ii) if $J$ and $L$ are both orthogonal to $K$, then $J \cap(K \oplus L)=J \cap L$.

Let $M$ and $N$ be non-trivial topologically complementary subspaces of a Hilbert space $\mathcal{H}$. Clearly

$$
\mathcal{H}=\left(M \cap N^{\perp}\right) \oplus\left(M^{\perp} \cap N\right) \oplus R
$$

where $R=\left(\left(M \cap N^{\perp}\right) \oplus\left(M^{\perp} \cap N\right)\right)^{\perp}$. By the above lemma, since $M$ and $R$ are both orthogonal to $M^{\perp} \cap N$, we have $M \cap\left(\left(M^{\perp} \cap N\right) \oplus R\right)=M \cap R$. So, again by using the lemma,

$$
\begin{aligned}
M & =M \cap\left(\left(M \cap N^{\perp}\right)+\left(\left(M^{\perp} \cap N\right) \oplus R\right)\right) \\
& =M \cap N^{\perp}+M \cap\left(\left(M^{\perp} \cap N\right) \oplus R\right) \\
& =M \cap N^{\perp}+M \cap R .
\end{aligned}
$$

Define $M_{o}$ by $M_{o}=M \cap R$. Then $M=\left(M \cap N^{\perp}\right) \oplus M_{o}$. Similarly we have $N=\left(M^{\perp} \cap N\right) \oplus N_{o}$, where $N_{o}$ is defined by $N_{o}=N \cap R$. 
Lemma 2. The subspaces $M_{o}$ and $N_{o}$ (as defined immediately above) are in generic position in $R$.

Proof. We must show that $M_{o} \cap N_{o}=M_{o}^{p} \cap N_{o}^{p}=M_{o}^{p} \cap N_{o}=M_{o} \cap N_{o}^{p}=(0)$, where $M_{o}^{p}=R \ominus M_{o}$ and $N_{o}^{p}=R \ominus N_{o}$. Clearly

$$
M_{o}^{\perp}=M^{\perp} \vee R^{\perp}=M^{\perp} \vee\left(M \cap N^{\perp}\right) \vee\left(M^{\perp} \cap N\right)=\left(M \cap N^{\perp}\right) \oplus M^{\perp},
$$

and similarly $N_{o}^{\perp}=\left(M^{\perp} \cap N\right) \oplus N^{\perp}$. Using Lemma 1 (ii) now gives

$$
M_{o}^{p}=R \cap M_{o}^{\perp}=R \cap\left(\left(M \cap N^{\perp}\right) \oplus M^{\perp}\right)=R \cap M^{\perp} .
$$

Similarly $N_{o}^{p}=R \cap N^{\perp}$. Since $M \cap N=M^{\perp} \cap N^{\perp}=(0)$, we have $M_{o} \cap N_{o}=M \cap$ $N \cap R=(0)$ and $M_{o}^{p} \cap N_{o}^{p}=M^{\perp} \cap N^{\perp} \cap R=(0)$. Also, $M_{o}^{p} \cap N_{o}=R \cap M^{\perp} \cap N=(0)$ and $M_{o} \cap N_{o}^{p}=R \cap M \cap N^{\perp}=(0)$.

Next we describe, up to unitary equivalence, arbitrary pairs of topologically complementary subspaces.

Theorem 1. Let $M$ and $N$ be non-trivial toplogically complementary subspaces of a Hilbert space $\mathcal{H}$. There exist Hilbert spaces $H$ and $K$, with $K$ possibly (0), and a positive injective contraction $A \in \mathcal{B}(H)$ such that the pair of subspaces $\{M, N\}$ of $\mathcal{H}$ is unitarily equivalent to the pair of subspaces $\{G(-A) \oplus K, G(A) \oplus(0)\}$ of $H \oplus H \oplus K$.

Proof. The Hilbert space $\mathcal{H}$ decomposes as $\mathcal{H}=\left(M \cap N^{\perp}\right) \oplus\left(M^{\perp} \cap N\right) \oplus R$, and following this decomposition $M=\left(M \cap N^{\perp}\right) \oplus(0) \oplus M_{o}$ and $N=(0) \oplus\left(M^{\perp} \cap N\right) \oplus$ $N_{o}$, where $M_{o}=M \cap R$ and $N_{o}=N \cap R$. Without loss of generality we can suppose that $\operatorname{dim}\left(M^{\perp} \cap N\right) \leq \operatorname{dim}\left(M \cap N^{\perp}\right)$. If $\operatorname{dim}\left(M^{\perp} \cap N\right)<\operatorname{dim}\left(M \cap N^{\perp}\right)$, let $H_{1}$ be a subspace of $M \cap N^{\perp}$ satisfying $\operatorname{dim} H_{1}=\operatorname{dim}\left(M^{\perp} \cap N\right)$. Otherwise, let $H_{1}=M \cap N^{\perp}$. Let $K$ be the orthogonal complement of $H_{1}$ in $M \cap N^{\perp}$, so that $M \cap N^{\perp}=H_{1} \oplus K$ (and $K$ is possibly (0)). Thus

$$
\begin{aligned}
\mathcal{H} & =H_{1} \oplus K \oplus\left(M^{\perp} \cap N\right) \oplus R, \\
M & =H_{1} \oplus K \oplus(0) \oplus M_{o}, \\
\text { and } N & =(0) \oplus(0) \oplus\left(M^{\perp} \cap N\right) \oplus N_{o} .
\end{aligned}
$$

By the preceding lemma, $M_{o}$ and $N_{o}$ are in generic position in $R$, so by Halmos' result [2], the pair $\left\langle M_{o}, N_{o}\right\rangle$ is unitarily equivalent to a pair of subspaces $\langle G(-B), G(B)\rangle$ of $H_{2} \oplus H_{2}$, for some Hilbert space $H_{2}$ and some positive injective contraction $B \in \mathcal{B}\left(H_{2}\right)$ with $I-B$ injective. Also, since $H_{1}$ and $M^{\perp} \cap N$ have the same dimensions and are orthogonal, the pair $\left\langle H_{1}, M^{\perp} \cap N\right\rangle$ is unitarily equivalent to $\langle G(-I), G(I)\rangle$, where $I$ is the identity operator on $H_{1}$. It follows that $\mathcal{H}, M$ and $N$ are unitarily equivalent, respectively, to

$$
\begin{aligned}
\mathcal{H}^{\prime} & =\left(H_{1} \oplus H_{1}\right) \oplus\left(H_{2} \oplus H_{2}\right) \oplus K, \\
M^{\prime} & =G(-I) \oplus G(-B) \oplus K, \\
\text { and } N^{\prime} & =G(I) \oplus G(B) \oplus(0),
\end{aligned}
$$

and so to

$$
\begin{aligned}
\mathcal{H}^{\prime \prime} & =H \oplus H \oplus K, \\
M^{\prime \prime} & =G(-A) \oplus K, \\
\text { and } N^{\prime \prime} & =G(A) \oplus(0),
\end{aligned}
$$


where $H=H_{1} \oplus H_{2}$ and $A \in \mathcal{B}(H)$ is a positive injective contraction given by $A=\left(\begin{array}{cc}I & 0 \\ 0 & B\end{array}\right)$.

Remarks. 1. In the above proof, if $M$ and $N$ are in generalised generic position, then $K=(0)$, and a proof of Papadakis' observation easily results.

2. Given $H, K$ and $A \in \mathcal{B}(H)$ as in the statement of the preceding theorem, it is easy to verify directly (even if $A$ is not a contraction) that the subspaces $\mathcal{M}=G(-A) \oplus K$ and $\mathcal{N}=G(A) \oplus(0)$ are topologically complementary and

(i) are orthogonal if and only if $A=I$,

(ii) are in generalised generic position if and only if $K=(0)$,

(iii) are in generic position if and only if $K=(0)$ and $I-A$ is injective.

Notice that $\mathcal{M}^{\perp} \cap \mathcal{N}=\{(A x, x, 0): x \in \operatorname{ker}(I-A)\}$ and $\mathcal{M} \cap \mathcal{N}^{\perp}=$ $\{(x,-A x, y): x \in \operatorname{ker}(I-A)$ and $y \in K\}$.

Lemma 3. Let $\left\{M_{1}, N_{1}\right\}$ and $\left\{M_{2}, N_{2}\right\}$ be non-trivial algebraically complementary pairs of subspaces of Hilbert spaces $H_{1}$ and $H_{2}$, respectively. If $\operatorname{dim} M_{1}=\operatorname{dim} M_{2}$ and $\operatorname{dim} N_{1}=\operatorname{dim} N_{2}$, then $\left\langle M_{1}, N_{1}\right\rangle$ is similar to $\left\langle M_{2}, N_{2}\right\rangle$.

Proof. Let $\operatorname{dim} M_{1}=\operatorname{dim} M_{2}$ and $\operatorname{dim} N_{1}=\operatorname{dim} N_{2}$. Then there exist unitary operators $U_{1}: M_{1} \rightarrow M_{2}$ and $U_{2}: N_{1} \rightarrow N_{2}$. Let $P$ be the projection onto $M_{1}$ along $N_{1}$ and define $T: H_{1} \rightarrow H_{2}$ by $T x=U_{1} P x+U_{2}(I-P) x$. This operator clearly has the desired properties.

Theorem 2. Let $H$ and $K$ be non-zero separable Hilbert spaces and let $A \in \mathcal{B}(H)$ be a postive injective operator. The pair of non-trivial topologically complementary subspaces $\{G(-A) \oplus K, G(A) \oplus(0)\}$ of $H \oplus H \oplus K$ is similar to a pair of the form $\{G(X), G(Y)\}$, where $X, Y \in \mathcal{B}\left(H_{1}\right)$ and $H_{1}$ is a Hilbert space, if and only if $A$ is non-compact.

Proof. Suppose first that $\{G(-A) \oplus K, G(A) \oplus(0)\}$ is similar to a pair of the form $\{G(X), G(Y)\}$ on $H_{1} \oplus H_{1}$, and assume, to the contrary, that $A$ is compact. Then $\{G(X), G(Y)\}$ is a pair of non-trivial topologically complementary subspaces of $H_{1} \oplus H_{1}$, and $\langle G(X), G(Y)\rangle$ is similar to the pair $\langle G(0), G(Y-X)\rangle$ (by the map $\left.\left(\begin{array}{cc}I & 0 \\ -X & I\end{array}\right)\right)$ and the latter pair are in generic position, as clearly

$$
\left((0) \oplus H_{1}\right) \cap G(Y-X)=(0) \quad \text { and } \quad G(0) \cap G(Y-X)^{\perp}=(0)
$$

since

$$
\left.G(Y-X)^{\perp}=\left\{\left(\left(X^{*}-Y^{*}\right) x, x\right): x \in H_{1}\right\}\right) .
$$

By Halmos' result, $\langle G(0), G(Y-X)\rangle$ is similar (even unitarily equivalent) to $\langle G(-B), G(B)\rangle$ for some positive injective contraction $B$ on a Hilbert space $H_{2}$. Thus, composing maps, there exists an invertible operator $S: H \oplus H \oplus K \rightarrow H_{2} \oplus H_{2}$ mapping the pair $\{G(-A) \oplus K, G(A) \oplus(0)\}$ onto $\{G(-B), G(B)\}$. In particular, since $\operatorname{dim} G(-B)=\operatorname{dim} G(B)=\operatorname{dim} H_{2}$ and $\operatorname{dim} G(A) \oplus(0)=\operatorname{dim} H$, it follows that $\operatorname{dim} H_{2}=\operatorname{dim} H$ and we can suppose that $H_{2}=H$. Let

$$
\left(\begin{array}{lll}
S_{11} & S_{12} & S_{13} \\
S_{21} & S_{22} & S_{23}
\end{array}\right)
$$

be the matrix of $S$. For every $x \in H, S(x, A x, 0)=\left(\left(S_{11}+S_{12} A\right) x,\left(S_{21}+S_{22} A\right) x\right)$; so, since $S$ maps $G(A) \oplus(0)$ bijectively onto $G(-B)$ or $G(B)$, it follows that $S_{11}+$ $S_{12} A$ is an invertible operator on $H$. Similarly, since for every $x \in H$ and $y \in$ 
$K$ we have $S(x,-A x, y)=\left(\left(S_{11}-S_{12} A\right) x+S_{13} y,\left(S_{21}-S_{22} A\right) x+S_{23} y\right)$, the operator $T: H \oplus K \rightarrow H$ given by $T(x, y)=\left(S_{11}-S_{12} A\right) x+S_{13} y$ is invertible. Thus, $T(H \oplus(0)) \neq H$, so the operator $S_{11}-S_{12} A$ on $H$ is not invertible. But $S_{11}-S_{12} A=\left(S_{11}+S_{12} A\right)-2 S_{12} A$, where $S_{11}+S_{12} A$ is invertible and $-2 S_{12} A$ is compact. By Weyl's theorem (see [10, page 7]) $S_{11}-S_{12} A$ is not injective. This contradicts the injectivity of $S$, and so $A$ must be a non-compact operator.

Conversely, suppose that $A$ is non-compact. Then, of course, $H$ must be infinitedimensional. In fact, by an elementary application of the spectral theorem, $H$ decomposes as $H=H_{1} \oplus H_{2}$ with $H_{1}$ infinite-dimensional and with $A=\left(\begin{array}{cc}A_{1} & 0 \\ 0 & A_{2}\end{array}\right)$ relative to this decomposition, with $A_{1} \in \mathcal{B}\left(H_{1}\right)$ an invertible operator. By Lemma 3 there exists an invertible operator $T: H_{1} \oplus H_{1} \oplus K \rightarrow H_{1} \oplus H_{1}$ such that

$$
T\left(G\left(-A_{1}\right) \oplus K\right)=G\left(-A_{1}\right) \quad \text { and } \quad T\left(G\left(A_{1}\right) \oplus(0)\right)=G\left(A_{1}\right) .
$$

Define $\tilde{T}:\left(H_{1} \oplus H_{1} \oplus K\right) \oplus\left(H_{2} \oplus H_{2}\right) \rightarrow\left(H_{1} \oplus H_{1}\right) \oplus\left(H_{2} \oplus H_{2}\right)$ by $\tilde{T}=\left(\begin{array}{cc}T & 0 \\ 0 & I\end{array}\right)$. Clearly $\tilde{T}$ is invertible. Let $U: H_{1} \oplus H_{1} \oplus H_{2} \oplus H_{2} \rightarrow H_{1} \oplus H_{2} \oplus H_{1} \oplus H_{2}$ and $V: H_{1} \oplus H_{1} \oplus K \oplus H_{2} \oplus H_{2} \rightarrow H_{1} \oplus H_{2} \oplus H_{1} \oplus H_{2} \oplus K$ be the unitary operators given by

$$
U=\left(\begin{array}{cccc}
I_{1} & 0 & 0 & 0 \\
0 & 0 & I_{2} & 0 \\
0 & I_{1} & 0 & 0 \\
0 & 0 & 0 & I_{2}
\end{array}\right), \quad V=\left(\begin{array}{ccccc}
I_{1} & 0 & 0 & 0 & 0 \\
0 & 0 & 0 & I_{2} & 0 \\
0 & I_{1} & 0 & 0 & 0 \\
0 & 0 & 0 & 0 & I_{2} \\
0 & 0 & I_{K} & 0 & 0
\end{array}\right)
$$

where $I_{1}, I_{2}$ and $I_{K}$ are the identity operators on $H_{1}, H_{2}$ and $K$, respectively. Define the invertible operator $S: H_{1} \oplus H_{2} \oplus H_{1} \oplus H_{2} \oplus K \rightarrow H_{1} \oplus H_{2} \oplus H_{1} \oplus H_{2}$ by $S=U \tilde{T} V^{-1}$. Then $S(G(-A) \oplus K)=G(-A)$ and $S(G(A) \oplus(0))=G(A)$. This completes the proof.

The preceding theorem raises the question: Which pairs of non-trivial topologically complementary subspaces of Hilbert space are similar to pairs of the form $\{G(X), G(Y)\}$ where $X$ and $Y$ are operators on some Hilbert space? The following proposition provides a geometric characterisation of this. In its proof, the proof that (ii) implies (iii) is due to H. K. Middleton [8, Corollary 1.3]. It is published here for the first time, with the author's permission.

Proposition 1. Let $\{M, N\}$ be a pair of non-trivial topologically complementary subspaces of a Hilbert space $H$. The following are equivalent:

(i) $\{M, N\}$ is similar to a pair of subspaces in generic position,

(ii) $M$ and $N$ have a common algebraic complement,

(iii) $\{M, N\}$ is similar to a pair of the form $\{G(X), G(Y)\}$, where $X$ and $Y$ are operators on some Hilbert space.

Proof. (i) $\Rightarrow$ (ii): Suppose that $\{M, N\}$ is similar to a pair of subspaces in generic position. Then, using Halmos' result and composing maps, $\{M, N\}$ is similar to a pair of the form $\{G(-A), G(A)\}$ on some Hilbert space $H_{1}$, with $A \in \mathcal{B}\left(H_{1}\right)$. Since the subspace (0) $\oplus H_{1}$ is a common algebraic complement of $G(-A)$ and $G(A), M$ and $N$ also have a common algebraic complement.

(ii) $\Rightarrow$ (iii): Suppose that $M$ and $N$ have a common algebraic complement, $L$ say. 
Then $M \cap L=N \cap L=(0)$ and $M+L=N+L=H$. If $x \in H$ and $P_{M}\left(I-P_{N}\right) x=0$, then $\left(I-P_{N}\right) x \in M^{\perp} \cap N^{\perp}=(0)$, so $x \in N$. Conversely, if $x \in N$, then $P_{M}\left(I-P_{N}\right) x=0$. Hence $\operatorname{ker}\left(P_{M}\left(I-P_{N}\right)\right)=N$, and it follows that the operator $\left(I-P_{N}\right)_{\mid M}: M \rightarrow N^{\perp}$ has dense range (its range is $\mathcal{R}\left(\left(I-P_{N}\right) P_{M}\right)$, whose closure is $\left.\operatorname{ker}\left(P_{M}\left(I-P_{N}\right)\right)^{\perp}=N^{\perp}\right)$. Since the operator $\left(I-P_{N}\right)_{\mid M}$ is also injective, it follows that $\operatorname{dim} M=\operatorname{dim} N^{\perp}$ (see [4, Problem 42]). Similarly $\operatorname{dim} L=\operatorname{dim} N^{\perp}$, so $\operatorname{dim} M=\operatorname{dim} L$. Let $U: L \rightarrow M$ be a unitary map, and let $P$ be the projection onto $M$ along $L$. Define the operator $S: H \rightarrow M \oplus M$ by $S x=(P x, U(I-P) x)$. Then $S$ is invertible and $S(M)=M \oplus(0), S(L)=(0) \oplus M$.

Let $z \in M$. Then $z=y+x$ with $y \in L$ and $x \in N$. We have $z=P z=P y+P x=$ $P x$. Thus, for every $z \in M$, there exists $x \in N$ such that $z=P x$. This $x \in N$ is unique. For if $z=P x_{1}=P x_{2}$ with $x_{1}, x_{2} \in N$, then $x_{1}-x_{2} \in N \cap L=(0)$. Define the map $T: M \rightarrow M$ by $T z=U(I-P) x$, where $x \in N$ and $z=P x$. Then $T$ is linear and since $S(N)=G(T), T$ is bounded by the Closed Graph Theorem. This shows that $\{M, N\}$ is similar to $\{G(0), G(T)\}$.

(iii) $\Rightarrow$ (i): Let $\{M, N\}$ be similar to $\{G(X), G(Y)\}$, where $X$ and $Y$ are operators on some Hilbert space. Now $\{G(X), G(Y)\}$ is similar to $\{G(0), G(Y-X)\}$ (by the invertible map $\left.\left(\begin{array}{cc}I & 0 \\ -X & I\end{array}\right)\right)$ and the latter pair are in generic position.

This completes the proof.

Remarks. 1. If $H, K$ and $A$ are as in the statement of Theorem 2 and $A$ is compact, Proposition 1 shows that the pair of subspaces $\{G(-A) \oplus K, G(A) \oplus$ $(0)\}$ cannot have a common algebraic complement. This fact is not easy to prove directly.

2. A subspace lattice is said to be medial if every pair of non-trivial elements of it are topologically complementary. A medial subspace lattice with precisely three non-trivial elements is called a double triangle. If $\left\{T_{\gamma}\right\}_{\gamma \in \Gamma}$ is a family of operators, with at least two elements, on a Hilbert space $H$ such that, for every $\alpha \neq \beta$, the operator $T_{\alpha}-T_{\beta}$ is injective with dense range, then $\{(0), H \oplus H\} \cup$ $\left\{G\left(T_{\gamma}\right): \gamma \in \Gamma\right\}$ is a medial subspace lattice on $H \oplus H$. Such medial subspace lattices, where each non-trivial element is the graph of some operator, will be called operator medial. A minor extension of the proof of the equivalence of (ii) and (iii) in the preceding proposition gives the following generalisation: A medial subspace lattice, with at least two non-trivial elements, on a Hilbert space is similar to an operator medial subspace lattice if and only if its nontrivial elements have a common algebraic complement. The latter result, for the case where the medial subspace lattice is a double triangle, was first proved in [8].

3. Let $\mathcal{T}=\{(0), L, M, N, H\}$ be a double triangle subspace lattice on a Hilbert space $H$, in which each of the vector sums $L+M, M+N$ and $N+L$ is closed (for example, let $H$ be finite-dimensional). Then $\mathcal{T}$ is similar to the double triangle $\mathcal{T}_{1}=\{(0),(0) \oplus M, M \oplus(0), G(I), M \oplus M\}$ (this was first observed in [8]) and to the operator double triangle $\mathcal{T}_{2}=\{(0), G(-I), G(0), G(I), M \oplus$ $M\}$. To see this, note that since $L$ is a common algebraic complement of $M$ and $N$, the proof of the implication (ii) $\Rightarrow($ iii) in Proposition 1 shows that $\langle L, M, N\rangle$ is similar to $\langle(0) \oplus M, M \oplus(0), G(T)\rangle$, where $T \in \mathcal{B}(M)$. Since $(M \oplus(0)) \cap G(T)=(0), T$ is injective; and since $(M \oplus(0))+G(T)=M \oplus M$, 
$T$ is surjective. Thus $T$ is invertible. Clearly $\langle(0) \oplus M, M \oplus(0), G(T)\rangle$ is similar to $\langle(0) \oplus M, M \oplus(0), G(I)\rangle$ (by the invertible map $\left(\begin{array}{cc}I & 0 \\ 0 & T^{-1}\end{array}\right)$ ). But it is also similar to $\langle G(-I), G(0), G(I)\rangle$ by the invertible map $\left(\begin{array}{cc}2 & -T^{-1} \\ 0 & T^{-1}\end{array}\right)$ (whose inverse is $\left(\begin{array}{cc}\frac{1}{2} & \frac{1}{2} \\ 0 & T\end{array}\right)$ ).

The previously mentioned example, due to T. Kato, of a double triangle which is not similar to any operator double triangle answered a question raised in [7]. It has the form $\{(0), H \oplus(0),(0) \oplus H, G(T), H \oplus H\}$, where $T$ is an unbounded linear transformation. We present a second example below which involves only compact operators. For some other results on double triangles and further references, see [3], $[5],[6],[7]$.

Example. Let $H$ be a separable infinite-dimensional Hilbert space, and let $A \in$ $\mathcal{B}(H)$ be positive injective and compact. By a result of von Neumann $[1$, Theorem 3.6] there exists a positive injective compact operator $B \in \mathcal{B}(H)$ such that $\mathcal{R}(A) \cap \mathcal{R}(B)=(0)$. Since $A+B$ is positive injective and compact, the same result shows that there exists a positive injective compact operator $C \in \mathcal{B}(H)$ such that $\mathcal{R}(A+B) \cap \mathcal{R}(C)=(0)$. Let $K$ be any non-zero subspace of $H$ and let $L$ be the subspace of $H \oplus H \oplus K$ given by $L=\{(x, B x+C y, y): x \in H$ and $y \in K\}$. Let $M=G(-A) \oplus K$ and $N=G(A) \oplus(0)$. It is easily verified that

$$
L \cap M=M \cap N=N \cap L=L^{\perp} \cap M^{\perp}=M^{\perp} \cap N^{\perp}=N^{\perp} \cap L^{\perp}=(0) .
$$

For example, if $x \in H, y \in K$ and $(x,-A x, y) \in L$, then $-A x=B x+C y$. Thus $C y=-(A+B) x \in \mathcal{R}(A+B) \cap \mathcal{R}(C)=(0)$, so $x=y=0$. Therefore $L \cap M=(0)$. Also, $L^{\perp}=\left\{\left(-B x, x,-P_{K} C x\right): x \in H\right\}$ and $N^{\perp}=\{(-A x, x, y): x \in H$ and $y \in$ $K\}$. If $x \in H$ and $\left(-B x, x,-P_{K} C x\right) \in N^{\perp}$, then $-B x=-A x \in \mathcal{R}(A) \cap \mathcal{R}(B)=$ (0), so $x=0$. This shows that $N^{\perp} \cap L^{\perp}=(0)$. The double triangle subspace lattice $\mathcal{T}=\{(0), L, M, N, H \oplus H \oplus K\}$ is not similar to an operator double triangle (on a Hilbert space). For if it were, the pair $\{M, N\}$ would then be similar to a pair of the form $\{G(X), G(Y)\}$ on $H_{1} \oplus H_{1}$, where $X, Y \in \mathcal{B}\left(H_{1}\right)$ and $H_{1}$ is a Hilbert space. This would contradict Theorem 2 .

\section{REFERENCES}

[1] P. A. Fillmore and J. P. Williams, On operator ranges, Adv. in Math. 7 (1971), 254-281. MR 45:2518

[2] P. R. Halmos, Two subspaces, Trans. Amer. Math. Soc. 144 (1969), 381-389. MR 40:4746

[3] P. R. Halmos, Reflexive lattices of subspaces, J. London Math. Soc.4 (1971), 257-263. MR 44:5808

[4] P. R. Halmos, A Hilbert space problem book, Van Nostrand, Princeton N. J., 1967. MR 34:8178

[5] E. Kissin, On some reflexive lattices of subspaces, J. Oper. Theory (1) 25 (1991), 141-162. MR 94f: 47006

[6] M. S. Lambrou and W. E. Longstaff, Finite rank operators leaving double triangles invariant, J. London Math. Soc. (2) 45 (1992), 153-168. MR 93e:47056

[7] W. E. Longstaff, Non-reflexive double triangles, J. Austral. Math. Soc. Ser. A 35 (1983), 349-356. MR 85c:47006

[8] H. K. Middleton, On the reflexivity and transitvity of non-distributive subspace lattices, Ph.D. Thesis, University of Western Australia, 1988. 
[9] M. Papadakis, On isomorphisms between certain non-CSL algebras, Proc. Amer. Math. Soc. 119 (1993), 1157-1164. MR 94g:47058

[10] H. Radjavi and P. Rosenthal, Invariant Subspaces, Springer-Verlag, Berlin and New York, 1973. MR 51:3924

Department of Mathematics, The University of Western Australia, Nedlands, Western Australia 6907, Australia

E-mail address: longstaff@maths.uwa.edu.au

E-mail address: oreste@maths.uwa.edu.au 